ISSN 0103-9954

\title{
INFLUENCIAS DE LA DENSIDAD Y PODAS SOBRE LA PRODUCCIÓN DE Pinus taeda L. A LOS 7 AÑOS DE EDAD.
}

\section{STAND DENSITY AND PRUNE EFFECTS ON THE YIELD OF A SEVEN YEAR OLD Pinus taeda L. STAND}

\author{
Rubén Costas ${ }^{1}$ Patricio Mac Donagh ${ }^{2}$ Elizabeth Weber ${ }^{3}$ \\ Santiago Figueredo ${ }^{4}$ Pedro Irschick ${ }^{4}$
}

\section{RESUMEN}

En un ensayo con 3 niveles de densidad y 12 tratamientos de poda, se evaluaron los efectos de la densidad, la altura de poda, el número de levantes de poda y la interacción entre la densidad y las podas sobre la producción de la masa de Pinus taeda L. a los 7 años de edad. Las variables evaluadas fueron el diámetro a la altura de pecho medio (DAP), la altura media, el volumen individual, el volumen por unidad de superficie, el área basal, el diámetro máximo sobre muñón (DMSM), el coeficiente de forma de Girard (CFG), el diámetro de ramas (DRBC) y el ángulo de inserción de las ramas (ARBC) en la base de la copa. En los tratamientos con mayor densidad de plantación se observaron mayores volúmenes por unidad de superficie, área basal y CFG que en los tratamientos con menor densidad. Estos produjeron mayores DAP, volúmenes individuales, DMSM, DRBC y ARBC que los tratamientos con mayores densidades. Los tratamientos con menores alturas finales de poda produjeron mayores DAP, volúmenes individuales, área basal y volúmenes por unidad de superficie que los tratamientos con mayores alturas de poda. Los menores DMSM se obtuvieron con alturas finales de poda de 3,30 m y 4,40 m realizadas a los 4 años de edad y los mayores DMSM ocurrieron al no efectuarse podas a los 5 años hasta $5,50 \mathrm{~m}$ y $6,60 \mathrm{~m}$. No se encontraron diferencias significativas entre los valores medios de DRBC, ARBC y CFG producidos por los 12 tratamientos de poda. La interacción entre densidad y los tratamientos de poda no fue significativa para ninguna de las variables respuestas.

Palabras clave: Pinus; diámetro a la altura de pecho; volumen; conicidad.

\section{ABSTRACT}

In an experiment with 3 stand densities and 12 prune treatments there were analyzed the effects of stand density, prune and interaction between them on the yield of a Pinus taeda L. plantation. The stand variables analyzed were diameter at breast height (DBH), stem volume, volume per hectare, height, basal area, diameter over stubs (DMSM), Girard form coefficient (CFG), diameter of branch (DRBC) and branche's angle (ARBC) at crown base. In the treatments with high density there were observed higher volume per hectare, basal area, CFG and lower DBH, stem volume, DMSM, DRBC and ARBC. In the treatments with lower prune height there were observed higher DBH, stem volume, volume per hectare, basal area than the treatments with larger prune height. Lower DMSM was observed with final prune height of $3,30 \mathrm{~m}$ and $4,40 \mathrm{~m}$ made at the age of 4 year. Higher DMSM were observed with final prune height of 5,5 and $6,6 \mathrm{~m}$ made at the age of 5 year. No statiscaly significant effects were found of the prune treatments over $\mathrm{DRBC}, \mathrm{ARBC}$ and CFG or interaction between stand density and prune height. The density and prune yield effects were independent from each other.

Key words: Pinus; diameter at breast height; volume; taper.

\section{INTRODUCCION}

La densidad de plantación y las podas efectuadas influyen sobre la cantidad y calidad de la

1. Ingeniero Forestal Instituto Subtropical de Investigaciones Forestales, Facultad de Ciencias Forestales, UNaM. Bertoni 124, Eldorado, Misiones, Argentina. CP 3380.rcostas@facfor.unam.edu.ar

2. Ingeniero Forestal Instituto Subtropical de Investigaciones Forestales, Facultad de Ciencias Forestales, UNaM. Bertoni 124, Eldorado, Misiones, Argentina. CP 3380.mdonagh@facfor.unam.edu.ar

3. Ingeniero Forestal Instituto Subtropical de Investigaciones Forestales, Facultad de Ciencias Forestales, UNaM. Bertoni 124, Eldorado, Misiones, Argentina. CP 3380. eweber@facfor.unam.edu.ar

4. Ingeniero Forestal Instituto Subtropical de Investigaciones Forestales, Facultad de Ciencias Forestales, UNaM. Bertoni 124, Misiones, Argentina. CP 3380.

Recebido para publicação em 4/05/2004 e aceito em 16/08/2005. 
producción de una masa forestal. Densidades altas posibilitan mayor producción total por unidad de superficie y menor producción individual que densidades de plantación bajas.

Las podas realizadas en edades tempranas permiten obtener trozas basales con nudos circunscriptos a un cilindro central de diámetro reducido que aumentan el valor económico de la masa al realizarse la cosecha.

En distintos ensayos de Pinus taeda, Fahler et al. (1986) para edad de plantación 8 años, Crechi et al. (1992) para edades entre 9 y 19 años y Costas et al. (2002 y 2003) para edades entre 5 y 6 años, encontraron que menores densidades de plantación inicial produjeron mayores diámetros a la altura de pecho medios y menores volúmenes por unidad de superficie y área basal.

En su revisión sobre la influencia de la densidad sobre la producción de Pinus radiata, Mason (2000), citó trabajos que indicaron que la conicidad, los diámetros de ramas y los ángulos de inserción de ramas fueron mayores en bajas densidades.

Meneses y Guzman (2000), al analizar una muestra de 30 rodales de Pinus radiata con distinta historia de raleos y podas, concluyeron que para alcanzar valores altos de índices de trozas podadas deben realizarse podas tempranas y frecuentes, un primer raleo junto con las podas y otro a los 10 años de edad. Seitz (1995) citó los resultados de un experimento de poda de Pinus taeda, donde fueron aplicados tratamientos con distintas proporciones de eliminación de copa verde a los 4 años de edad. Al evaluarse los efectos de los tratamientos sobre los árboles dominantes al cabo de un año, se encontró que el incremento de DAP fue prácticamente nulo con poda total de copa, alcanzando el máximo al no haberse eliminado ramas. Para el mismo período de tiempo, no hubo diferencias de incrementos de altura en función de los tratamientos aplicados.

Fassola et al. (2002), tomando muestras aleatorias de un ensayo con 4 niveles de densidad producidos por raleos, 4 niveles de intensidad de poda y 3 tratamientos de realces de poda en Pinus taeda, encontraron que el DAP, la altura y el volumen fueron afectadas en distinto grado por la intensidad de los raleos y de las podas.

El valor de la madera libre de nudos en el mercado mundial es altamente superior que la madera con nudos. A fines del año 2003, aserraderos del norte de Misiones, Argentina, pagaron precios entre 30\% y $60 \%$ superiores por trozas podadas respecto del precio de trozas sin poda de diámetros en punta fina superiores a $35 \mathrm{~cm}$. La inserción de la producción forestal del nordeste argentino en el mercado maderero internacional potencia la importancia económica de la realización de podas oportunas.

Los objetivos del presente trabajo fueron evaluar los efectos de la densidad, la altura de poda, el número de levantes de poda y la interacción entre densidad y podas sobre variables silvícolas explicativas de la producción de Pinus taeda a los 7 años de edad.

\section{MATERIALES Y MÉTODOS}

El material experimental es una masa regular de Pinus taeda Origen Marion, implantada en 1996 en la localidad de Garuhapé-mí, Dto. Gral. Libertador Gral. San Martín, Misiones, Argentina. Las coordenadas geográficas de ubicación del ensayo son $26^{\circ} 50^{\prime} 00^{\prime \prime}$ de latitud Sur y $54^{\circ} 32^{\prime} 30^{\prime \prime}$ de longitud Oeste. La superficie total del mismo es de $19.080 \mathrm{~m}^{2}$. La pendiente promedio del lote es de 3\%. El suelo es homogéneo en todo el ensayo y corresponde a un Kandiudult típico (Soil Taxonomy, 1991). El material es homogéneo respecto de la procedencia genética, sitio y tratamientos culturales realizados hasta los 3 años de edad. A esa edad se realizó una poda de aproximadamente $1,8 \mathrm{~m}$ de altura sobre toda la masa y sobre dos tercios de la plantación fueron realizados raleos sistemáticos, producto de los cuales un tercio quedó con aproximadamente el $38 \%$ y el otro tercio raleado con aproximadamente el $58 \%$ de los árboles de la plantación original. La densidad de cada tercera parte fue controlada hasta llegando a los 6 años de edad a los valores mostrados en la Tabla 1.

En cada bloque con densidad homogénea, fueron aplicados al azar los 12 tratamientos de poda mostrados en la Tabla 1. Los 3 niveles de densidad, 4 niveles de altura final de poda y 3 niveles de número de levantes de poda conformaron un ensayo factorial con 36 combinaciones, y dos repeticiones para cada una. Según la clasificación de Seitz (1995), se adoptó el criterio de altura de poda fija a todos los árboles de 
cada unidad experimental. Las alturas medias a los 4, 5 y 6 años de edad fueron 7,23 y 9,29 10,84 m y los porcentajes máximos de copa removida a esas edades fueron 47,$88 ; 64,11$ y $35,64 \%$ respectivamente.

En cada parcela a los 7 años de edad se midieron los DAP de todos los árboles. Las mediciones de altura total, diámetros de ramas en el verticilo inferior de la copa (DRBC), diámetro del fuste a 5,27 m desde el suelo y la estimación visual del ángulo de inserción de las ramas (ARBC) en la base de la copa fueron realizadas en cuatro árboles por parcela: el de mayor DAP, el de menor DAP y los 2 árboles con DAP más próximos al valor mediano. En la Figura 1 se muestra un esquema de las variables medidas o estimadas en cada uno de estos 4 árboles. Los valores de los diámetros máximos sobre muñones (DMSM) fueron obtenidos de los registros del mismo ensayo desde los cuatro y hasta los seis años de edad, que fueron las edades en las cuales se aplicaron los distintos tratamientos de podas.

A los 288 pares de valores (DAP, h) observados se ajustó la función $h=b_{0}+b_{1} \ln (D A P)$ citada por Prodan et al. (1997), obteniéndose la ecuación $h=2,7449+3,3506 * \ln (D A P)$, con la cual fueron predichas las alturas no medidas de los árboles de cada parcela. El coeficiente de determinación del ajuste fue 0,35 .

Previamente al análisis estadístico, se verificó la homogeneidad de calidad de sitio entre los bloques con densidad diferente comparando las alturas dominantes. El análisis estadístico fue realizado a través de análisis de variancia de un ensayo factorial de un modelo lineal de efectos fijos, evaluando simultáneamente los efectos de la densidad, de las alturas finales de poda, del número de levantes de poda y de la interacción entre dichos factores. Además se realizaron pruebas de comparaciones de pares de medias utilizando la prueba de Tukey. En todos los tests se consideró un nivel de significación del $5 \%$ para identificar diferencias estadísticamente significativas entre las medias involucradas en cada prueba.

En todos los análisis de variancia se comprobó el cumplimiento de los supuestos de homogeneidad de variancia y distribución normal de los errores. Cuando se encontraron apartamientos leves de uno o ambos supuestos, se optó por presentar los resultados de los análisis con las observaciones sin transformar. Las pruebas realizadas con modelos de efectos fijos como los aplicados en este ensayo son robustas en caso de apartamientos leves de los supuestos (Montgomery, 1991).

En el análisis de algunas de las variables de respuesta se incorporó como covariables los valores medios de cada una de ellas a la edad de 4 años, a los efectos de mejorar la sensibilidad de las pruebas realizadas y explicar los resultados obtenidos a los 7 años de edad.

Todo el perímetro del ensayo y cada bloque con la respectiva densidad está rodeado de líneas de bordura de la misma plantación, sobre las cuales no se realizó ninguna medición y tienen la función de evitar la influencia de un nivel de densidad sobre otro.

Las variables respuestas analizadas y sus unidades de medida fueron las siguientes:

a) Diámetro a la altura de pecho medio $(\mathrm{cm})$.

b) Altura promedio (m).

c) Volumen individual medio $\left(\mathrm{dm}^{3}\right)$. El volumen total para cada árbol fue calculado utilizando la función:

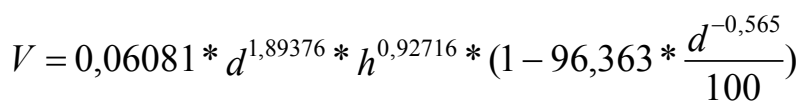
del árbol (m).

Donde: $\mathrm{V}$ es el volumen total sin corteza $\left(\mathrm{dm}^{3}\right), \mathrm{d}$ es el diámetro a la altura de pecho $(\mathrm{cm}) \mathrm{y}$ h es la altura total

d) Area Basal $\left(\mathrm{m}^{2} / \mathrm{ha}\right)$.

e) Volumen total por unidad de superficie $\left(\mathrm{m}^{3} / \mathrm{ha}\right)$.

f) Diámetro máximo sobre muñón $(\mathrm{cm})$

g) Coeficiente de forma de Girard modificado $-\mathrm{CFG}=\mathrm{d}_{5,27} / \mathrm{d}$

Donde $\mathrm{d}_{5,27}$ : diámetro con corteza producido a 5,27 metros desde el suelo.

h) Diámetro de ramas en la base de la copa $(\mathrm{cm})$.

i) Ángulo de inserción de las ramas en la base de la copa (grados sexagesimales). 
TABLA 1: Estructura de Tratamientos de densidad y de poda.

TABLE 1: Stand density and prune treatments.

\begin{tabular}{c|c|c|c|c|c}
\hline $\begin{array}{c}\text { Densidad 6 } \\
\text { (arboles/ha) }\end{array}$ & $\begin{array}{c}\text { Levantes de } \\
\text { poda }\end{array}$ & $\begin{array}{c}\text { Altura poda } \\
\text { año 4 }(\mathrm{m})\end{array}$ & $\begin{array}{c}\text { Altura poda } \\
\text { año 5 }(\mathrm{m})\end{array}$ & $\begin{array}{c}\text { Altura poda } \\
\text { año 6 (m) }\end{array}$ & $\begin{array}{c}\text { Tratamiento } \\
\text { de poda }\end{array}$ \\
\hline & 2 & 3,30 & 3,30 & 3,30 & 1 \\
& 3 & 2,60 & 3,30 & 3,30 & 2 \\
& 4 & 2,37 & 2,83 & 3,30 & 3 \\
& 2 & 4,40 & 4,40 & 4,40 & 4 \\
D1=400 & 3 & 3,15 & 4,40 & 4,40 & 5 \\
D2=700 & 4 & 2,73 & 3,56 & 4,40 & 6 \\
D3=1000 & 2 & 1,80 & 5,50 & 5,50 & 7 \\
& 3 & 3,70 & 3,70 & 5,50 & 8 \\
& 4 & 3,10 & 4,30 & 5,50 & 9 \\
& 2 & 1,80 & 6,60 & 6,60 & 10 \\
& 3 & 4,25 & 4,25 & 6,60 & 11 \\
& 4 & 3,47 & 5,03 & 6,60 & 12 \\
\hline
\end{tabular}

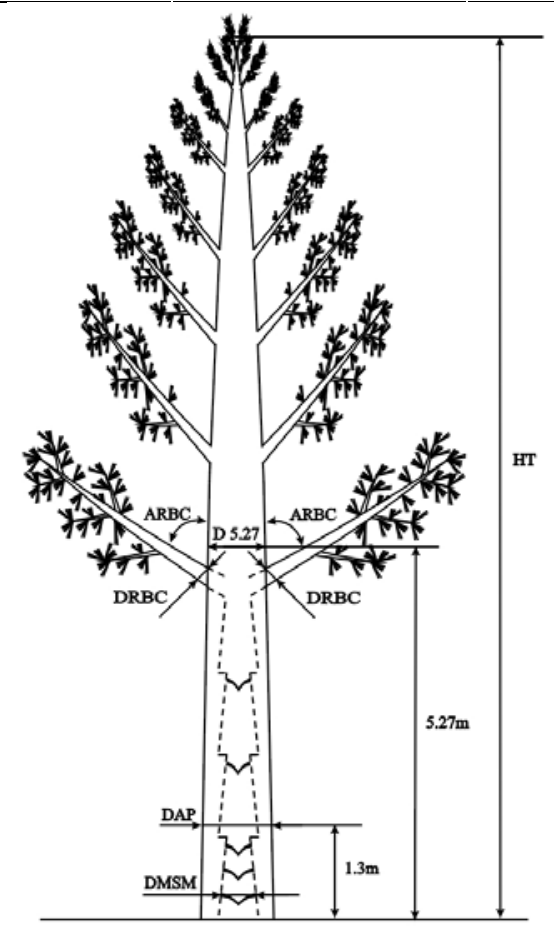

Figura 1: Variables medidas o estimadas en cada árbol.

Figure 1: Variables measured and mated in each three.

\section{RESULTADOS Y DISCUSION}

\section{Diámetro a la altura de pecho medio (DAP)}

Se encontraron diferencias significativas entre las medias de DAP debidas al efecto de la densidad. Los resultados son mostrados en la Tabla 2. La prueba de Tukey indicó que la media en la densidad D1 fue significativamente superior a la producida en la densidad D2 y ésta a su vez mayor a la obtenida en la densidad D3.

Los DAP medios producidos en dos de los tres niveles de menor altura final de poda (tratamientos 2 y 3) resultaron ser estadísticamente superiores a los DAP medios en cuatro de los seis niveles de mayor altura final de poda (tratamientos 8, 9, 10 y 11). Debido al tiempo reducido desde la finalización de la aplicación de algunos de los tratamientos de poda, es probable que sus efectos hayan quedado confundidos con los valores iniciales de cada unidad experimental indicadas por la covariable DAP4. 
No se encontraron diferencias significativas de DAP entre los tratamientos con 2, 3 y 4 levantes de poda. La comprobación del cumplimiento de los supuestos mostró homogeneidad de variancia y leve apartamiento de la distribución normal de los residuales.

TABLA 2: DAP medios en cada nivel de densidad y cada tratamiento de poda.

TABLE 2: Average DBH in each density and in each prune treatment.

\begin{tabular}{c|c|c|c|c|c}
\hline \multicolumn{3}{c|}{$\begin{array}{c}\text { Efectos de la densidad } \\
\mathrm{F}(2,35)=18,12^{*}\end{array}$} & \multicolumn{3}{c}{$\begin{array}{c}\text { Efectos de la poda } \\
\mathrm{F}(11,35)=5,17^{*}\end{array}$} \\
\hline $\begin{array}{c}\text { Densidad } \\
(\mathrm{arb} / \mathrm{ha})\end{array}$ & $\begin{array}{c}\text { DAP } \\
(\mathrm{cm})\end{array}$ & $\begin{array}{c}\text { DAP4 } \\
(\mathrm{cm})\end{array}$ & Tratamiento poda & $\begin{array}{c}\text { DAP } \\
(\mathrm{cm})\end{array}$ & $\begin{array}{c}\text { DAP4 } \\
(\mathrm{cm})\end{array}$ \\
\hline 400 & $20,86^{\mathrm{a}}$ & 11,63 & 2 & $20,97^{\mathrm{a}}$ & 11,47 \\
700 & $19,28^{\mathrm{b}}$ & 11,52 & 3 & $20,84^{\mathrm{a}}$ & 11,22 \\
1000 & $17,70^{\mathrm{c}}$ & 10,04 & 5 & $19,73^{\mathrm{ab}}$ & 11,02 \\
\hline La interacción entre la densidad y los & 4 & $19,63^{\mathrm{ab}}$ & 11,29 \\
tratamientos de podas no fue significativa & 7 & $19,59^{\mathrm{abc}}$ & 11,80 \\
respecto del DAP. & & 1 & $19,46^{\mathrm{abc}}$ & 10,89 \\
& & 12 & $19,34^{\mathrm{abc}}$ & 11,32 \\
& & 9 & $18,91^{\mathrm{bc}}$ & 11,27 \\
& & 10 & $18,65^{\mathrm{bc}}$ & 11,35 \\
& & 6 & $18,21^{\mathrm{bc}}$ & 10,03 \\
& & 8 & $18,17^{\mathrm{bc}}$ & 10,11 \\
& & 11 & $17,82^{\mathrm{c}}$ & 10,98 \\
\hline
\end{tabular}

Donde: Tratamientos identificados con letras iguales no tuvieron diferencias significativas dado $\alpha=0,05$; * Valores del estadístico F que indican diferencias significativas entre tratamientos con $\alpha=0,05$; DAP $4=$ diámetro a la altura del pecho medio en cada tratamiento a los 4 años.

\section{Altura media (ALT)}

La altura media no fue afectada por la densidad, por las alturas finales de poda ni por el número de levantes de poda. En la Tabla 3 se muestran los promedios de ALT en cada densidad. La comprobación de supuestos arrojó que los residuales tuvieron distribución aproximadamente normal y variancia estadísticamente homogénea.

TABLA 3: Promedios de ALT en cada nivel de densidad.

TABLE 3: Average ALT in each density.

\begin{tabular}{cc}
\hline Densidad (arb/ha) & ALT (m) \\
\hline 1000 & $12,56^{\mathrm{a}}$ \\
700 & $12,55^{\mathrm{a}}$ \\
400 & $12,35^{\mathrm{a}}$ \\
\hline
\end{tabular}

Donde: Tratamientos identificados con letras iguales no tuvieron diferencias significativas entre sí, dado $\alpha=0,05$.

\section{Volumen individual promedio (vi)}

La prueba de Tukey indicó que el promedio de vi en la densidad D1 resultó significativamente superior al de la densidad D2 y éste a su vez superior al producido en la de densidad D3.

Se encontraron diferencias de vi debido al efecto de las podas. Como se puede observar en la Tabla 4 , dos de los tres tratamientos de menor altura final de poda produjeron vi estadísticamente mayores que cinco de los seis tratamientos de mayor altura de poda.

No se encontraron diferencias significativas entre los tratamientos con distinta cantidad de levantes de poda. Se notaron leves apartamientos de los supuestos de homogeneidad de variancia y distribución normal de los residuales. 
TABLA 4: Promedios de vi en cada nivel de densidad y cada tratamiento de poda.

TABLE 4: Average stem volume in each density and in each prune treatment.

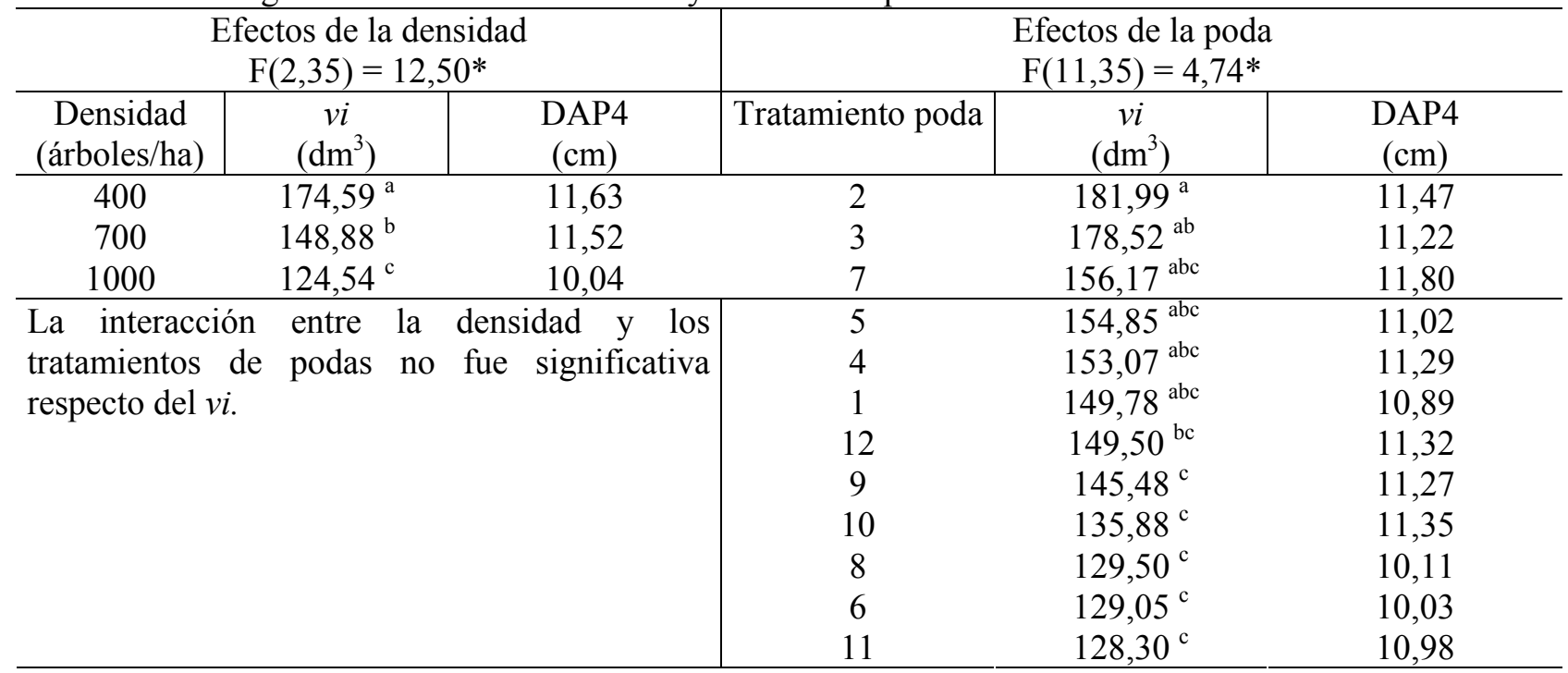

Donde: Tratamientos identificados con letras iguales no tuvieron diferencias significativas entre sí, dado $\alpha=0,05 ; *=$ Valores del estadístico F que indican diferencias significativas entre tratamientos con $\alpha=0,05$; DAP4 $=$ diámetro a la altura del pecho medio en cada tratamiento a $\operatorname{los} 4$ años.

\section{Area basal (G)}

En la mayor densidad el $\mathrm{G}$ fue significativamente superior al de la densidad intermedia y ésta a su vez superior a la producida en la densidad menor (Tabla 5).

En general las mayores $G$ se observaron en tratamientos con menores alturas finales de poda, destacándose las diferencias significativas entre los tratamientos 2 y 3 respecto de los tratamientos 10 y 11 . Los dos primeros fueron los de menor altura final con más realces de podas y los dos últimos fueron los de mayor altura final de poda con menor cantidad de levantes.

No fueron significativas las diferencias de $\mathrm{G}$ debidas al efecto de los distintos números de levantes de poda. Se cumplieron los supuestos de homogeneidad de variancia y distribución normal de los residuales. TABLA 5: Areas basales promedios en cada nivel de densidad y cada tratamiento de poda.

TABLE 5: Average basal area in each density and in each prune treatment.

\begin{tabular}{c|c|c|c|c|c}
\hline \multicolumn{3}{c|}{$\begin{array}{c}\text { Efectos de la densidad } \\
\mathrm{F}(2,35)=18.16^{*}\end{array}$} & \multicolumn{3}{c}{$\mathrm{F}(11,35)=2,65^{*}$} \\
\hline Densidad & $\mathrm{G}$ & $\mathrm{G} 4\left(\mathrm{~m}^{2} / \mathrm{ha}\right)$ & Tratamiento & $\mathrm{G}$ & $\mathrm{G} 4$ \\
\hline 1000 & $25,70^{\mathrm{a}}$ & 12,38 & 3 & $24,19^{\mathrm{a}}$ & 9,41 \\
700 & $20,92^{\mathrm{b}}$ & 7,71 & 2 & $23,02^{\mathrm{ab}}$ & 9,08 \\
400 & $14,14^{\mathrm{c}}$ & 5,36 & 7 & $21,51^{\mathrm{abc}}$ & 8,75 \\
\hline La interacción entre la densidad y los & 1 & $21,36^{\mathrm{abc}}$ & 9,14 \\
tratamientos de podas no fue significativa & 5 & $21,00^{\mathrm{abc}}$ & 9,09 \\
respecto del G. & & & 4 & $20,58^{\mathrm{abc}}$ & 9,73 \\
& & & 9 & $20,13^{\mathrm{abc}}$ & 9,23 \\
& & & 10 & $19,54^{\mathrm{bc}}$ & 8,23 \\
& & & 11 & $18,58^{\mathrm{c}}$ & 8,43 \\
& & & 6 & $17,84^{\mathrm{c}}$ & 6,70 \\
& & & $17,82^{\mathrm{c}}$ & 7,51 \\
\hline
\end{tabular}

Donde: Tratamientos identificados con letras iguales no tuvieron diferencias significativas entre sí, dado $\alpha=0,05 ; *=$ Valores del estadístico $\mathrm{F}$ que indican diferencias significativas entre tratamientos con $\alpha=0,05 ; \mathrm{G} 4=$ área basal promedio en cada tratamiento a los 4 años.

\section{Volumen por unidad de superficie (V)}

Se encontraron diferencias significativas entre las medias de $\mathrm{V}$ debidas al efecto de la densidad. La 
prueba de Tukey indicó que la media en la densidad D3 fue significativamente superior a la producida en la densidad D2 y ésta a su vez mayor que el V medio de la densidad D1 (Tabla 6).

TABLA 6: Volúmenes por hectárea promedios en cada nivel de densidad y tratamiento de poda. TABLE 6: Volume per hectare results in each density and prune treatment.

\begin{tabular}{c|c|c|c|c|c}
\hline \multicolumn{3}{c}{$\begin{array}{c}\text { Efectos de densidad } \\
\mathrm{F}(2,35)=15,75^{*}\end{array}$} & \multicolumn{3}{c}{$\begin{array}{c}\text { Efectos de la poda } \\
\mathrm{F}(11,35)=2,75^{*}\end{array}$} \\
\hline $\begin{array}{c}\text { Densidad } \\
\text { (arboles/ha) }\end{array}$ & $\begin{array}{c}\mathrm{V} \\
\left(\mathrm{m}^{3} / \mathrm{ha}\right)\end{array}$ & $\begin{array}{c}\mathrm{V} 4 \\
\left(\mathrm{~m}^{3} / \mathrm{ha}\right)\end{array}$ & $\begin{array}{c}\text { Tratamiento } \\
\text { poda }\end{array}$ & $\begin{array}{c}\mathrm{V} \\
\left(\mathrm{m}^{3} / \mathrm{ha}\right)\end{array}$ & $\begin{array}{c}\mathrm{V} 4 \\
\left(\mathrm{~m}^{3} / \mathrm{ha}\right)\end{array}$ \\
\hline 1000 & $126,17^{\mathrm{a}}$ & 35,48 & 3 & $120,99^{\mathrm{a}}$ & 27,63 \\
700 & $103,04^{\mathrm{b}}$ & 22,00 & 2 & $116,09^{\mathrm{ab}}$ & 26,03 \\
400 & $70,11^{\mathrm{c}}$ & 15,22 & 7 & $106,94^{\mathrm{abc}}$ & 24,57 \\
\hline La interacción entre la densidad y los tratamientos & 1 & $105,04^{\text {abcd }}$ & 27,16 \\
de podas no fue significativa respecto del V. & 5 & $103,00^{\text {abcd }}$ & 25,73 \\
\multicolumn{1}{l}{} & 12 & $102,06^{\text {abcd }}$ & 28,29 \\
& 4 & $99,86^{\text {abcd }}$ & 26,27 \\
& 9 & $96,62^{\mathrm{bcd}}$ & 23,22 \\
& & 10 & $89,88^{\mathrm{cd}}$ & 23,81 \\
& & 8 & $87,37^{\mathrm{cd}}$ & 21,80 \\
& & 6 & $85,75^{\mathrm{cd}}$ & 17,90 \\
\end{tabular}

Donde: Tratamientos identificados con letras iguales no tuvieron diferencias significativas entre sí, dado $\alpha=0,05 ; *=$ Valores del estadístico $\mathrm{F}$ que indican diferencias significativas entre tratamientos con $\alpha=0,05$; V4 = volumen por hectárea promedio en cada tratamiento a los 4 años.

Si bien en general se produjeron mayores $\mathrm{V}$ al aplicarse menores alturas de poda finales, al realizarse la prueba de Tukey las diferencias no resultaron explicadas claramente por los tratamientos de poda. No se encontraron diferencias significativas entre los tratamientos con distinta cantidad de levantes de poda. Se notaron leves apartamientos de los supuestos de homogeneidad de variancia y distribución normal de los residuales.

Los resultados de los análisis del efecto de la densidad sobre DAP medio, altura media, área basal y volumen por unidad de superficie a los 7 años de edad fueron similares a los obtenidos por Fahler et al. (1986) con un ensayo de 8 años de edad y por Crechi et al. (1992) para edades de 9 a 19 años, exceptuando los resultados y conclusiones de estos últimos respecto de la altura media. Se mantuvieron las tendencias mostradas por Costas et al. (2002 y 2003) para el mismo ensayo a los 5 y 6 años de edad.

\section{Diámetro máximo sobre muñón (DMSM)}

Los dos tratamientos de menor densidad produjeron DMSM estadísticamente homogéneos entre sí y superiores al de la mayor densidad (Tabla 7).

Los tratamientos de poda 3, 7 y 10, los de menores alturas de poda en el cuarto año de edad produjeron los mayores DMSM, resultaron estadísticamente homogéneos entre sí y superiores a los tratamientos 1, 4, 8 y 11. Los tratamientos 1 y 4 fueron los de altura final de poda lograda a los cuatro años de edad y los tratamientos 8 y 11 fueron los de mayor altura de poda realizada a esa edad. La segunda poda correspondiente a los tratamientos 7 y 10 se realizó recién al $5^{\circ}$ año de edad debido a que su aplicación en el $4^{\circ}$ año implicaba llevar la poda a más de $5,50 \mathrm{~m}$ y $6,60 \mathrm{~m}$ respectivamente, eliminando más del $66 \%$ de longitud de copa verde. Se notó en esos tratamientos el impacto negativo de la demora de las podas, dado que produjo 2 de los 3 mayores DMSM.

Aunque el número de levantes de poda no tuvo efectos significativos, sí resultó significativa su interacción con la altura final de poda (HFP). La Figura 2 muestra comportamientos diferentes del DMSM al llevarse la poda a las 4 alturas finales según cuántos realces se llevaron a cabo. Con 3 y 4 levantes de poda el DMSM tendió a ser mayor a menores alturas de poda finales. Con sólo 2 podas aplicadas, los mayores DMSM se dieron en las mayores alturas finales de poda, efectuadas un año después de las logradas en las dos menores alturas de poda. El DMSM fue afectado más por la oportunidad de levante de poda que por la altura 
de poda. Las podas más altas realizadas tardía y drásticamente produjeron los mayores DMSM.

TABLA 7: DMSM promedios en cada nivel de densidad y tratamiento de poda.

TABLE 7: Average DMSM in each density and prune treatment.

\begin{tabular}{c|c|c}
\hline \multicolumn{2}{c}{$\begin{array}{c}\text { Efectos de la densidad } \\
\mathrm{F}(2,36)=23,59^{*}\end{array}$} & \multicolumn{2}{c}{$\begin{array}{c}\text { Efectos de la poda } \\
\mathrm{F}(11,36)=12,12^{*}\end{array}$} \\
\hline $\begin{array}{c}\text { Densidad } \\
\text { (arboles/ha) }\end{array}$ & $\begin{array}{c}\text { DMSM } \\
(\mathrm{cm})\end{array}$ & Tratamiento poda \\
$(\mathrm{cm})$
\end{tabular}

Donde: Tratamientos identificados con letras iguales no tuvieron diferencias significativas entre sí, dado $\alpha=0,05 ; *=$ Valores del estadístico $\mathrm{F}$ que indican diferencias significativas entre tratamientos con $\alpha=0,05$; DMSM $=$ diámetro máximo sobre muñón.

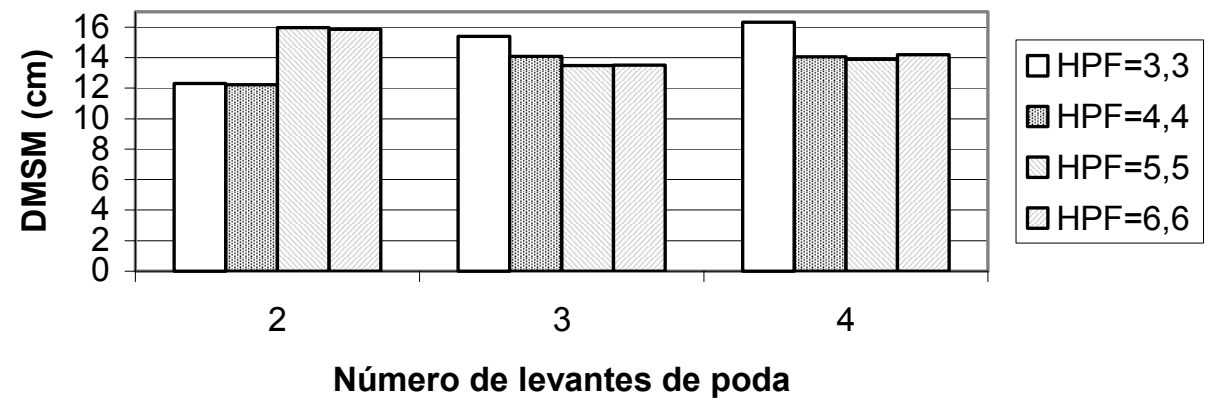

Figura 2: Diámetros máximos sobre muñón (DMSM) medios producidos en cada combinación de número de levantes de poda y alturas finales de poda (HPF).

Figure 2: Means of diameter over stubs (DMSM) for each combination of number of prune raising and last prune height (HPF).

\section{Coeficiente de forma de Girard (CFG), Diámetro de ramas en la base de copa (DRBC) y Ángulo de ramas en la base de copa (ARBC)}

Los resultados de CFG, DRBC y ARBC se muestran en la Tabla 8. La densidad tuvo efectos significativos sobre el CFG, coeficiente indicador de la conicidad del fuste. En la mayor densidad se manifestó el mayor CFG, estadísticamente superior a los producidos en las otras dos densidades. Los tratamientos de poda no produjeron CFG diferentes entre sí. Tampoco fueron significativas las diferencias entre los distintos números de levantes de poda ni ninguna interacción entre efectos principales.

Los diámetros de ramas en la base de copa medios producidos en las dos densidades menores fueron estadísticamente homogéneos entre sí y superiores al DRBC resultante en la mayor densidad. Si bien se encontraron diferencias de DRBC entre los tratamientos de poda, las mismas no se correspondieron con distintas alturas de poda. El número de levantes de poda y las interacciones entre factores principales no tuvieron efectos significativos sobre el DRBC. 
El ARBC medio producido en la menor densidad fue significativamente superior al dado en la mayor densidad. Con el tratamiento con densidad intermedia se obtuvo un ARBC medio sin diferencias significativas con los otros dos niveles de densidad. No hubo diferencias significativas de ARBC entre los tratamientos de poda. El número de levantes de poda y las interacciones entre factores principales no tuvieron efectos significativos sobre el ARBC.

TABLA 8: Promedios de CFG, DRBC y ARBC en cada nivel de densidad.

TABLE 8: Average of CFG, DRBC and ARBC in each density.

\begin{tabular}{c|c|c|c}
\hline \multirow{2}{*}{ Densidad (arboles/ha) } & $\mathrm{F}(2,36)=11,62^{*}$ & $\mathrm{~F}(2,36)=9,74^{*}$ & $\mathrm{~F}(2,36)=7,35^{*}$ \\
\cline { 2 - 4 } & $\mathrm{CFG}$ & DRBC $(\mathrm{cm})$ & ARBC $\left(^{\mathrm{o}}\right)$ \\
\hline 1000 & $0,78^{\mathrm{a}}$ & $2,58^{\mathrm{b}}$ & $53,26^{\mathrm{b}}$ \\
700 & $0,74^{\mathrm{b}}$ & $3,06^{\mathrm{a}}$ & $57,40^{\mathrm{ab}}$ \\
400 & $0,73^{\mathrm{b}}$ & $3,17^{\mathrm{a}}$ & $63,40^{\mathrm{a}}$ \\
\hline
\end{tabular}

Donde: Tratamientos identificados con letras iguales no tuvieron diferencias significativas entre sí, dado $\alpha=0,05 ; *=$ Valores del estadístico F que indican diferencias significativas entre tratamientos con $\alpha=0,05 ; \mathrm{CFG}=$ Coeficiente de forma de Girard; DRBC = Diámetro de ramas en la base de copa y ARBC = Ángulo de ramas en la base de copa.

De los resultados respecto del coeficiente de forma de Girard, diámetros de ramas y ángulos de ramas medidos en la base de copa, puede interpretarse que en las mayores densidades hubo menor disminución del diámetro del fuste entre 1,3 y 5,27 m de altura, diámetros de ramas en la base de copa menores y ramas menos horizontales que con densidades menores. Estos resultados son coincidentes con los descriptos por Mason (2000) en su revisión del impacto de la densidad de plantación sobre la producción de Pinus radiata.

El efecto de la densidad fue importante para explicar la variación de 8 de las 9 variables respuesta analizadas. Respecto de las dos variables que resultan de la agregación de los individuos de la masa, VHA y $\mathrm{G}$, sus valores medios fueron mayores en los niveles de mayor densidad y viceversa.

Los tratamientos 2 y 3 fueron los de menor altura final de poda lograda más tardíamente. Produjeron DAP, vi, V y G significativamente mayores que los obtenidos con los tratamientos 10 y 11 , los dos de mayor altura final de poda lograda en menos tiempo. El efecto de los tratamientos de poda no fue importante para explicar la variación de la altura media.

A los 7 años de edad se manifestaron las limitaciones del diámetro en caso de podas muy severas que Meneses y Guzman (2000) expresaron que ocurren a la edad de cosecha.

En la evaluación de todas las variables respuesta se pudo separar los efectos de la densidad y de las podas y además se observó que la interacción entre ambos factores no fue significativa. La densidad y los tratamientos de poda se comportaron en forma aditiva sobre todas las variables analizadas, o sea que produjeron efectos independientes hasta los 7 años de edad, manteniéndose lo encontrado por Costas et al. (2002 y 2003) para el mismo ensayo a los 5 y 6 años de edad respectivamente.

\section{CONCLUSIONES}

En mayor medida la densidad y en menor medida los tratamientos de poda influyeron sobre las variables analizadas, excepto la altura media. Los tratamientos de densidad produjeron resultados que diferencian la cantidad y calidad de los productos de una plantación. Con densidades altas se produjeron fustes más cilíndricos y pequeños, ramas con menores ángulos de inserción y diámetros menores, respecto de los resultados obtenidos con densidades bajas.

La masa forestal debe manejarse desde edades tempranas para lograr los objetivos de producción. Como el manejo de la densidad y la realización de podas son complementarios y producen resultados independientes, es necesario prestar atención oportuna sobre ambos a la vez para obtener una producción que cuantitativa y cualitativamente optimice la rentabilidad al momento de la cosecha.

En condiciones de material genético y calidad de sitio similares a las del ensayo evaluado, de todas las combinaciones de tratamientos de densidad y poda aplicadas en este ensayo, puede recomendarse una densidad de 700 árboles por hectárea entre los 4 y 7 años de edad y realización de podas como las efectuadas con el tratamiento n. 12: 1,80; 3,47; 5,03 y $6,60 \mathrm{~m}$ de altura de poda a los $3,4,5$ y 6 años de edad 
respectivamente. Esta combinación equilibró la producción total por unidad de superficie con el tamaño promedio de los árboles, generó valores intermedios de conicidad, diámetros de ramas y ángulos de inserción de ramas en la base de la copa y produjo un fuste podado de más de 6,60 $\mathrm{m}$ de largo con un DMSM promedio de $14,18 \mathrm{~cm}$.

\section{AGRADECIMIENTOS}

A los alumnos de Ingeniería Forestal de la Facultad de Ciencias Forestales Martín Vargas, Claudio Gómez, Raquel Winck, Rafaela Morales, Marisa Becker, Juan Heck e Iván Khalstorf por su participación ad honorem en la toma de datos y parcialmente en el procesamiento de los mismos.

Al personal de campo de la empresa del Ing. Pedro Irschick, por su colaboración en la aplicación de tratamientos y en el mantenimiento del ensayo.

\section{REFERENCIAS BIBLIOGRAFICAS}

COSTAS R.; MAC DONAGH, P.; WEBER, E.; IRSCHICK, P; PALAVECINO, J. Efectos de la Densidad de plantación y la altura de poda sobre la producción de Pinus taeda L. a los 5 años de edad. Revista Floresta, v. 33, n. 1, p. 79-97, 2003.

COSTAS, R.; MAC DONAGH, P.; WEBER, E.; IRSCHICK, P; PALAVECINO, J. Efectos de la Densidad de plantación y la altura de poda sobre la produccion de Pinus taeda L. a los 6 años de edad. (En prensa Revista Forestal Venezolana desde 2003).

CRECHI, E.; FRIEDL, R.; FERNÁNDEZ, R. Efecto de la densidad de plantación sobre el crecimiento de Pinus taeda L. JORNADAS SOBRE PINOS SUBTROPICALES, 1992, Eldorado. Actas...Eldorado, Argentina : Centro de Investigaciones y Experiencias Forestales, 1992. p. 92-110.

FAHLER, J. C.; DI LUCCA, C.; OROZCO, E.; RODRÍGUEZ, G.; GIMÉNEZ, S. Comportamiento de 10 diferentes densidades de plantación inicial en Pinus taeda a los 8 años de edad en suelos del complejo 9 (C.A.R.T.A.) en la provincia de Misiones. E. E. A. Misiones. INTA. Informe Téenico, n. 46, p.1-14,1986.

FASSOLA, H. E.; MOSCOVICH, F. A.; FERRERE, P.; RODRÍGUEZ, F. Evolución de las principales variables de árboles de Pinus taeda L. sometidos a diferentes tratamientos silviculturales en el nordeste de la provincia de Corrientes, Argentina. Ciência Florestal, v. 12, n.2, p. 51-60, 2002.

MENESES, M; GUZMÁN, S. Análisis de la eficiencia de la silvicultura destinada a la obtención de madera libre de nudos en plantaciones de pino radiata en Chile. Bosque, Valdivia, v. 21, n. 2, p. 85-93, 2000.

MASON E. G. A brief review of the impact of stand density on variables affecting radiata pine stand value. Disponible en: (http://www.fore.canterbury.ac.nz/euan/spacing/density3.htm) $>$ Acceso en febrero de 2004.

MONTGOMERY, D.C. Diseño y Análisis de Experimentos. México D. F.: Iberoamérica, 1991. 589 p.

PRODAN M.; PETERS, R.; COX, F.; REAL, P. Mensura Forestal. San José, Costa Rica: IICA-BMZ/GTZ, 1997. $561 \mathrm{p}$.

SEITZ, R. A. Manual da Poda de Espécies Arbóreas Florestais. Curitiba : FUPEF, 1995. 88p.

SOIL SURVEY STAFF. Keys to Soil Taxonomy. AID. USDA, 1991. 754p. 